\title{
Improved Multi B-Value Diffusion-Weighted MRI of the Body by Simultaneous Model Estimation and Image Reconstruction (SMEIR) ${ }^{\star}$
}

\author{
Moti Freiman $^{1}$, Onur Afacan ${ }^{1}$, Robert V. Mulkern ${ }^{2}$, and Simon K. Warfield ${ }^{1}$ \\ 1 Computational Radiology Laboratory, Boston Children's Hospital, Harvard \\ Medical School, MA, USA \\ 2 Department of Radiology, Boston Children's Hospital, Harvard Medical School, \\ MA, USA
}

\begin{abstract}
Diffusion-weighted MRI images acquired with multiple bvalues have the potential to improve diagnostic accuracy by increasing the conspicuity of lesions and inflammatory activity with background suppression. Unfortunately, the inherently low signal-to-noise ratio (SNR) of DW-MRI reduces enthusiasm for using these images for diagnostic purposes. Moreover, lengthy acquisition times limit our ability to improve the quality of multi b-value DW-MRI images by multiple excitations acquisition and signal averaging at each b-value. To offset these limitations, we propose the Simultaneous Model Estimation and Image Reconstruction (SMEIR) for DW-MRI, which substantially improves the quality of multi b-value DW-MRI images without increasing acquisition times. Our model introduces the physiological signal decay model of DW-MRI as a constraint in the reconstruction of the DW-MRI images. An in-vivo experiment using 6 low-quality DW-MRI datasets of a healthy subject showed that SMEIR reconstruction of low-quality data improved SNR by $55 \%$ in the liver and by $41 \%$ in the kidney without increasing acquisition times. We also demonstrated the clinical impact of our SMEIR reconstruction by increasing the conspicuity of inflamed bowel regions in DW-MRI of 12 patients with Crohn's disease. The contrast-to-noise ratio (CNR) of the inflamed regions in the SMEIR images was higher by $12.6 \%$ relative to $\mathrm{CNR}$ in the original DW-MRI images.
\end{abstract}

\section{Introduction}

Diffusion-weighted MRI (DW-MRI) of the body is a non-invasive imaging technique sensitive to the incoherent motion of water molecules inside the area of interest. This motion is characterized by a combination of a slow diffusion component associated primarily with the Brownian motion of water molecules, and a

\footnotetext{
* This investigation is supported in part by NIH grants R01 LM010033 R42 MH086984 R01 EB008015 UL1 TR000170 and by research grants from the Translational Research Program at Boston Children's Hospital. M.F is also supported in part by a Research Fellow Award from the Crohn's and Colitis Foundation of America.
} 
fast diffusion component associated primarily with the bulk motion of intravascular molecules in the micro-capillaries [8].

The signal in DW-MRI images decays as a function of the amount of incoherent motion present in the tissue and a diffusion-weighting parameter known as "b-value." The rapid DW-MRI signal decay in tissue with normal diffusion characteristics, combined with the reduced signal decay in regions of restricted diffusion, increases the conspicuity of abnormal regions in DW-MRI images acquired with sufficiently high b-value and aids in detecting abnormal regions.

Recent studies demonstrate the potential of sufficiently high b-value DW-MRI images to improve the detection rate of different types of carcinoma [45]11], focal hepatic lesions [6], and inflammatory activity in the bowel [9] without ionized radiation and/or exogenous contrast media.

Unfortunately, DW-MRI images have an inherently low signal-to-noise ratio (SNR), which reduces enthusiasm for using these images for diagnostic purposes. Koh and Collins [7] recommend acquiring the DW-MRI data with multiple excitations (i.e., 5 to 6 excitations) and using the averaged signal to improve DW-MRI image quality. However, acquiring multi b-value DW-MRI data with multiple excitations to achieve both sufficient image quality and sufficient information for quantiative assessment of fast and slow diffusion will substantially increase the overall acquisition time, thus making this method less suitable for routine clinical use.

A unique feature in DW-MRI images in particular, and in parametric imaging techniques in general, is the addition of a $4^{\text {th }}$ dimension to the control parameters in the acquired data. This $4^{\text {th }}$ parametric dimension, which is the diffusionweighting factor (b-value) at play in DW-MRI, can be exploited as an additional source of prior information that can be utilized in reconstructing images.

Several groups have suggested incorporating the $4^{\text {th }}$ parametric dimension as a constraint to increase the quality of reconstructed images in quantitative T1 and T2 images [1212. However, these models are difficult to optimize and have not been successfully applied to body DW-MRI reconstruction.

In this work, we reduce the number of excitations required to obtain multi bvalue DW-MRI images of the body with sufficient SNR by introducing a Bayesian model of the expected signal with the signal decay model utilized as the prior information. With this model, we are able to simultaneously obtain high-quality DW-MRI images for multiple b-values at once and estimate the signal decay model parameter values instead of generating estimates of each b-value image independently and without estimating the signal decay model parameter values.

We also introduce an efficient iterative solution based on the ExpectationMaximization framework through which we obtain high SNR DW-MRI images and parameter estimates with the "Simultaneous Model Estimation and Image Reconstruction" (SMEIR) solver, a novel approach that simultaneously estimates the intra-voxel incoherent motion signal decay model parameters and reconstructs high-quality DW-MRI images. In our experiments, we have shown substantial improvements in DW-MRI image quality. Specifically, our SMEIR reconstruction approach improves the SNR of 6 DW-MRI datasets of a healthy 
volunteer by $55 \%$ in the liver and by $41 \%$ in the kidney. We have also assessed clinical impact, namely, by demonstrating increased conspicuity of inflammatory bowel regions in a study cohort of 12 pediatric Crohn's disease patients. Our results show that SMEIR-reconstructed images have a contrast-to-noise ratio that is $12.6 \%$ higher than the CNR produced by the original DW-MRI data.

\section{Method}

\subsection{DW-MRI Reconstruction Model}

DW-MRI images have an inherently low SNR, which reduces enthusiasm for using these images for diagnostic purposes. Specifically, the SNR of DW-MRI images decreases as the b-values used to acquire the images increases. As a rule, the SNR of DW-MRI images obtained with high b-values is increased by acquiring the images with multiple excitations at each b-value. An SNR-optimized DW-MRI image is then reconstructed by solving a Maximum-Likelihood estimation problem for each b-value independently:

$$
\hat{S}_{i}=\underset{S_{i}}{\operatorname{argmin}} \sum_{j=1}^{M}\left(S_{i}-S_{i, j}^{\prime}\right)^{2}
$$

where $S_{i, j}^{\prime}$ is the observed signal at excitation $j$ with b-value $b_{i}, S_{i}$ is the unknown signal, and $M$ is the number of excitations. The solution is simply obtained by averaging the signal at the different excitations:

$$
\hat{S}_{i}=\frac{1}{M} \sum_{j=1}^{M} S_{i, j}^{\prime}
$$

Unfortunately, by obtaining images with $M$ as the number of excitations (NEX), we are only able to achieve a SNR increase of $\sqrt{M}$, the low rate of which requires acquisitions of long duration.

As an alternative, we propose to incorporate a prior knowledge on signal evolution in the b-value dimension to reconstruct the DW-MRI images by adding a regularization term to Eq. 1 .

$$
\hat{S}_{i}=\underset{S_{i}}{\operatorname{argmin}} \sum_{j=1}^{M}\left(S_{i}-S_{i, j}^{\prime}\right)^{2}+\alpha\left(S_{i}-f(\Theta, i)\right)^{2}
$$

Where $\alpha$ is the regularization weighting parameter, $f(\Theta, i)$ is the expected signal at b-value $b_{i}$ given the signal decay model parameters $\Theta$. In this work we used the intra-voxel incoherent motion (IVIM) model of DW-MRI signal decay proposed by Le Bihan et al. [8]. This model assumes a signal decay function of the form:

$$
f(\Theta, i)=S_{0}\left(f \exp \left(-b_{i}\left(D+D^{*}\right)\right)+(1-f)\left(\exp \left(-b_{i} D\right)\right)\right)
$$


Where $f(\Theta, i)$ is the expected signal at b-value $b_{i}, \Theta=\left\{s_{0}, f, D^{*}, D\right\}$ are the intra-voxel incoherent motion model parameters describing the baseline (i.e., without any diffusion effect) signal $\left(s_{0}\right)$; the fast-diffusion fraction $(f)$; the fastdiffusion coefficient $\left(D^{*}\right)$ characterizing primarily the bulk motion of intravascular molecules in the micro-capillaries; and the slow-diffusion coefficient $(D)$ characterizing primarily the Brownian motion of water molecules in the extra-cellular space.

Unfortunately, the expected signal is dependent on the parameters of the signal decay model (i.e., $\Theta$ ) which are unknown. Therefore, we cannot optimize Eq. 3 directly. Instead, we formulate the reconstruction problem as a simultaneous reconstruction of the DW-MRI images acquired with different b-values and an estimation of the signal decay model:

$$
[\hat{S}, \hat{\Theta}]=\underset{S, \Theta}{\operatorname{argmin}} \sum_{i=1}^{N} \sum_{j=1}^{M}\left(S_{i}-S_{i, j}^{\prime}\right)^{2}+\alpha \sum_{i=1}^{N}\left(S_{i}-f(\Theta, i)\right)^{2}
$$

where $S=\left\{S_{1}, \ldots, S_{N}\right\}$.

\subsection{Optimization Scheme}

We used an Expectation-Maximization-like approach to solve Eq. 5]by iteratively estimating the signal decay model parameters $\Theta$ given the current estimate of signal $S$ and then estimating signal $S$ given the current estimate of the model parameters $\Theta$. We describe these steps in detail next.

E-Step: Signal decay model $(\Theta)$ estimation:

The expected DW-MRI signal decay at each voxel is described by the intra-voxel incoherent motion model (Eq. 4). We used the spatially constrained incoherent motion signal decay model described by Freiman et al. 3. to robustly estimate the model parameters $\Theta$. Given the current estimate of the DW-MRI signal $S^{t}$, the estimate of the model parameters $\Theta^{t}$ was obtained by minimizing:

$$
\hat{\Theta}^{t}=\underset{\Theta^{t}}{\operatorname{argmin}} \sum_{i=1}^{N}\left(S_{i}^{t}-f\left(\Theta^{t}, i\right)\right)^{2}+\sum_{v_{p} \sim v_{q}} g\left(\Theta_{v_{p}}^{t}, \Theta_{v_{q}}^{t}\right)
$$

where $g(\cdot, \cdot)$ is the spatial constraint given by:

$$
g\left(\Theta_{v_{p}}^{t}, \Theta_{v_{q}}^{t}\right)=\alpha W\left|\Theta_{v_{p}}^{t}-\Theta_{v_{q}}^{t}\right|
$$

and $\alpha \geq 0$ is the spatial coupling factor; $W$ is a diagonal weighting matrix that accounts for the different scales of the parameters in $\Theta$; and $v_{p}, v_{q}$ are the neighboring voxels established by the employed neighborhood system utilized in this model. We then estimated the model parameters $\Theta$ by minimizing Eq. 6 using the "fusion bootstrap moves" combinatorial solver introduced by Freiman et al. [3]. 
M-Step: DW-MRI Signal $(S)$ reconstruction:

Given the current estimate of the DW-MRI signal decay model $\Theta^{t}$, we minimized Eq. [5 using the BOBYQA non-linear optimization algorithm [10] to get the next estimate of the signal $S^{t+1}$.

First, we initialized the algorithm with the acquired DW-MRI data as the current estimate of the signal; and second, using the acquired DW-MRI data and the current estimate of the model parameters, we iteratively alternated between estimating the model parameters from the current signal (Eq. 6) and estimating the true DW-MRI signal (Eq. 5) until the change in the estimated signal became negligible.

\section{Experimental Results}

\subsection{In-vivo Evaluation Using Healthy Control}

We conducted an in-vivo study with healthy control volunteer data to analyze the improvement in SNR achieved by using our SMEIR approach.

We acquired DW-MRI data of a healthy volunteer using a 1.5-T unit (Magnetom Avanto, Siemens Medical Solutions, Erlangen, Germany). We performed free-breathing single-shot echo-planar imaging using the following parameters: repetition/echo time $(\mathrm{TR} / \mathrm{TE})=6800 / 59 \mathrm{~ms}$; matrix size $=192 \times 156$; field of view $=300 \times 260 \mathrm{~mm}$; slice thickness $/$ gap $=5 \mathrm{~mm} / 0.5 \mathrm{~mm} ; 40$ axial slices; $7 \mathrm{~b}$ values $=0,50,100,200,400,600,800 \mathrm{~s} / \mathrm{mm}^{2}$ with 6 excitations (i.e. NEX=6). The acquisition time for each excitation was 3:30 min. with an overall acquisition time of $21 \mathrm{~min}$.

We generated high-quality images by averaging the data from the 6 excitations and 6 low-quality datasets - each one consisting of data acquired with 1 NEX. For each low-quality dataset, we reconstructed the images using our SMEIR reconstruction approach. We experimentally set the value of $\alpha$ in Eq. 5 to 0.01 . The average (std) running time required to reconstruct DW-MRI images of $256 \times 256 \times 40$ voxels on an 8 processors machine Intel ${ }^{\circledR}$ Xeon ${ }^{\circledR}$ at 2.40 $\mathrm{GHz}$ with cache size of $12 \mathrm{MB}$ and overall memory of $48 \mathrm{~GB}$ using the SMEIR reconstruction was 8:04 (2:35) min.

For purposes of evaluation, we defined 2 spherical regions of interest (ROI) in the liver and in the kidney, respectively. We defined SNR at each voxel as the average signal over the 6 low-quality datasets divided by the standard deviation of the signal over these datasets. We likewise calculated SNR for the b-value $=800$ $\mathrm{s} / \mathrm{mm}^{2}$ DW-MRI image for the raw low-quality datasets (RAW) and for the SMEIR-reconstructed datasets (SMEIR). Next, we averaged SNR for the RAW and SMEIR data, respectively, over the liver and kidney ROIs.

Fig. 1 presents a high b-value (i.e. $800 \mathrm{~s} / \mathrm{mm}^{2}$ ) image acquired in high-quality $(\mathrm{NEX}=6)$; in low-quality $(\mathrm{NEX}=1)$; in low-quality $(\mathrm{NEX}=1)$ combined with SMEIR reconstruction; and a bar-plot representation of SNR of low-quality data with and without SMEIR reconstruction. The average \pm std SNR of SMEIR data $(12.2 \pm 2.5$ in the liver and $11 \pm 2.5$ in the kidney) was higher than the SNR of the low-quality data (7.9 \pm 4.2 in the liver and $7.8 \pm 2.7$ in the kidney) - a difference 


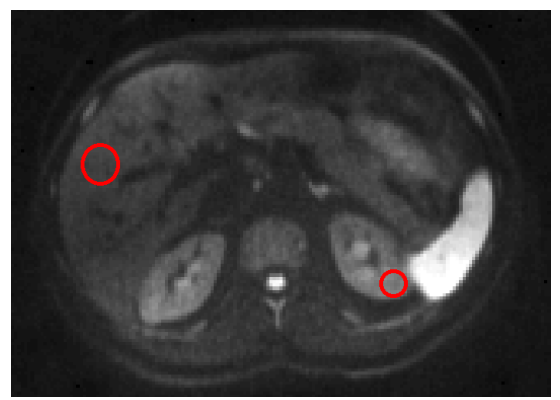

(a) High-quality DW-MRI (6 NEX)

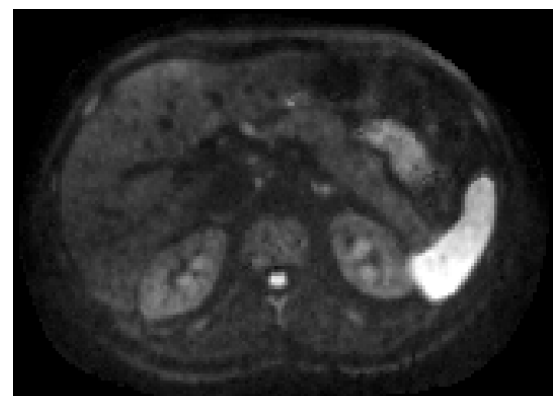

(c) SMEIR DW-MRI (1 NEX)

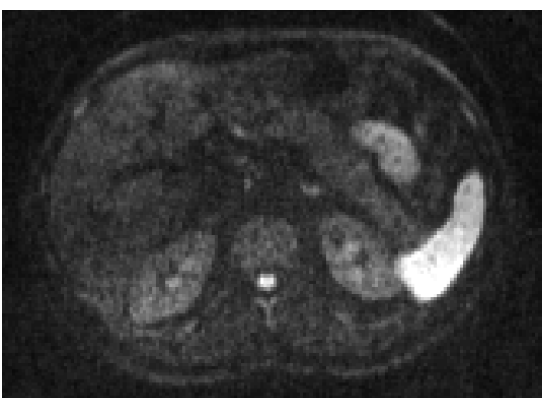

(b) Low-quality DW-MRI (1 NEX)

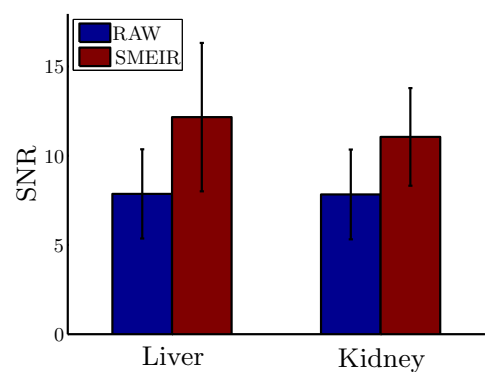

(d) Signal to Noise Ratio (SNR)

Fig. 1. In-vivo example. This figure presents $\mathrm{b}$-value $=800 \mathrm{~s} / \mathrm{mm}^{2}$ images acquired in high-quality $(\mathrm{NEX}=6)$; in low-quality $(\mathrm{NEX}=1)$; and in low-quality $(\mathrm{NEX}=1)$ combined with SMEIR reconstruction, and a bar-plot representation of SNR of low-quality data with and without SMEIR reconstruction.

that was statistically significant (Paired Student's t-test, $\mathrm{p}<0.0001$ ). Notably, the SMEIR reconstruction of low-quality data improved SNR by $55 \%$ in the liver and by $41 \%$ in the kidney without additional acquisition time.

\subsection{Clinical Impact}

To demonstrate the actual clinical impact of using our SMEIR reconstruction approach instead of the raw low-quality DW-MRI data, we assessed the conspicuity of inflamed bowel regions in $\mathrm{b}$-value $=800 \mathrm{~s} / \mathrm{mm}^{2}$ images by means of contrastto-noise ratio (CNR) between regions with active inflammation and surrounding normal tissues in DW-MRI data of Crohn's disease patients.

We retrospectively reviewed DW-MRI data of 30 patients who underwent clinical MRI exams including a MR enterography (MRE) protocol that included polyethylene glycol administration for bowel distention; gadolinium-enhanced, dynamic 3D VIBE (volume-interpolated breath hold exam); and DW-MRI with the same protocol described in section 3.1 acquired with 1 NEX. 


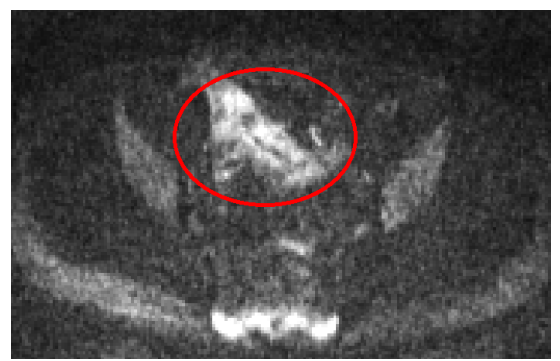

(a) Raw data

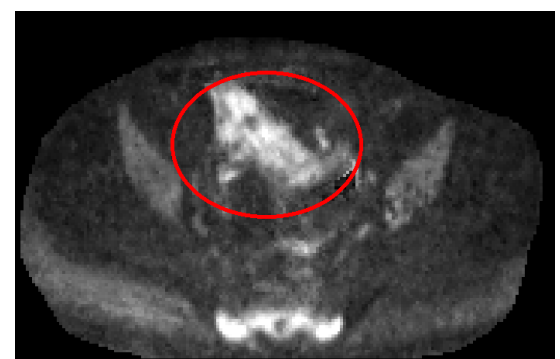

(b) SMEIR reconstruction

Fig. 2. Representative Crohn's disease patient with active inflammation in the ileum. (a) The acquired raw DW-MRI data; and (b) SMEIR-reconstructed data. The region with active inflammation is more conspicuous in the SMEIR-reconstructed image than in the raw DW-MRI data.

Two board-certified radiologists reviewed the MRE data independently. Disease activity was defined as abnormal bowel wall thickening and enhancement in the gadolinium-enhanced images by each of the readers. In case of disagreement between the two reviewers, consensus was reached by joint reading of the data. The consensus decision identified 12 patients with active inflammation in the ileum. Another board-certified radiologist, blinded to the MRE data and to the review, identified the ileum on the DW-MRI data for each patient. We manually annotated the ileum wall on the DW-MRI images with b-value $=200 \mathrm{~s} / \mathrm{mm}^{2}$. Next, we calculated the CNR of the inflamed ileum in b-value $=800 \mathrm{~s} / \mathrm{mm}^{2}$ images by subtracting the background signal from the signal of the inflamed ileum and dividing by the standard deviation of the signal in the ileum.

Fig. 2 depicts the acquired raw DW-MRI data and SMEIR-reconstructed data of a representative Crohn's disease patient with active inflammation in the ileum. Visually, the region with active inflammation is more conspicuous in the SMEIR-reconstructed image than in the raw DW-MRI data. Quantitatively, the average (std) CNR between the inflamed regions and the surrounding neighborhood in the SMEIR-reconstructed images was higher $(2.52 \pm 0.69)$ than in the raw DW-MRI data $(2.23 \pm 0.47)$ - a difference that was statistically significant (Paired Student's t-test, $\mathrm{p}<0.05)$. The SMEIR-reconstructed images improved CNR by $12.6 \%$.

\section{Conclusions}

We have presented a new model and method for reconstructing high-quality multi b-value DW-MRI images of the body without increasing overall acquisition times. This novel approach features the signal decay model as a prior knowledge in the image reconstruction, effectively enabling us to simultaneously reconstruct DW-MRI images and estimate the signal decay model parameters using the Expectation-Maximization framework. 
As demonstrated in our experiments, our method improves overall image quality by increasing the signal-to-noise ratio (SNR) by up to $55 \%$ and by increasing the conspicuity of inflamed bowel regions of pediatric Crohn's disease patients by $12.6 \%$ without increasing overall acquisition times. The proposed method permits the acquisition of high-quality DW-MRI images for diagnostic purposes within a clinically acceptable acquisition timeframe.

\section{References}

1. Block, K.T., Uecker, M., Frahm, J.: Model-based iterative reconstruction for radial fast spin-echo MRI. IEEE Trans. Med. Imaging 28(11), 1759-1769 (2009)

2. Doneva, M., Börnert, P., Eggers, H., Stehning, C., Sénégas, J., Mertins, A.: Compressed sensing reconstruction for magnetic resonance parameter mapping. Magn. Reson. Med. 64(4), 1114-1120 (2010)

3. Freiman, M., Voss, S.D., Mulkern, R.V., Perez-Rossello, J.M., Callahan, M.J., Warfield, S.K.: Reliable Assessment of Perfusivity and Diffusivity from Diffusion Imaging of the Body. In: Ayache, N., Delingette, H., Golland, P., Mori, K. (eds.) MICCAI 2012, Part I. LNCS, vol. 7510, pp. 1-9. Springer, Heidelberg (2012)

4. Ichikawa, T., Erturk, S.M., Motosugi, U., Sou, H., Iino, H., Araki, T., Fujii, H.: High-B-value diffusion-weighted MRI in colorectal cancer. AJR Am. J. Roentgenol. 187(1), 181-184 (2006)

5. Ichikawa, T., Erturk, S.M., Motosugi, U., Sou, H., Iino, H., Araki, T., Fujii, H.: High-b value diffusion-weighted MRI for detecting pancreatic adenocarcinoma: preliminary results. AJR Am. J. Roentgenol. 188(2), 409-414 (2007)

6. Kanematsu, M., Goshima, S., Watanabe, H., Kondo, H., Kawada, H., Noda, Y., Aomatsu, A., Moriyama, N.: Detection and characterization of focal hepatic lesions with diffusion-weighted MR imaging: a pictorial review. Abdom. Imaging (July 2012)

7. Koh, D.M., Collins, D.J.: Diffusion-weighted MRI in the body: applications and challenges in oncology. AJR Am. J. Roentgenol. 188(6), 1622-1635 (2007)

8. LeBihan, D., Breton, E., Lallemand, D., Aubin, M.L., Vignaud, J., Laval-Jeantet, M.: Separation of diffusion and perfusion in intravoxel incoherent motion MR imaging. Radiology 168(2), 497-505 (1988)

9. Oto, A., Kayhan, A., Williams, J.T.B., Fan, X., Yun, L., Arkani, S., Rubin, D.T.: Active Crohn's disease in the small bowel: evaluation by diffusion weighted imaging and quantitative dynamic contrast enhanced MR imaging. J. Magn. Reson. Imaging 33(3), 615-624 (2011)

10. Powell, M.: The BOBYQA algorithm for bound constrained optimization without derivatives. Technical report NA2009/06, Dep. App. Math. and Th. Physics, Cambridge, England (2009)

11. Sugita, R., Yamazaki, T., Furuta, A., Itoh, K., Fujita, N., Takahashi, S.: High b-value diffusion-weighted MRI for detecting gallbladder carcinoma: preliminary study and results. Eur. Radiol. 19(7), 1794-1798 (2009)

12. Velikina, J.V., Alexander, A.L., Samsonov, A.: Accelerating MR parameter mapping using sparsity-promoting regularization in parametric dimension. Magn. Reson. Med. (December 2012) (in press), http://dx.doi.org/10.1002/mrm.24577 